\title{
Shapes of RNA pseudoknot structures
}

\author{
Christian M. Reidys ${ }^{*} \dagger$ and Rita R. Wang * \\ * Center for Combinatorics, LPMC-TJKLC \\ $\dagger$ College of Life Sciences \\ Nankai University \\ Tianjin 300071 \\ P.R. China \\ Phone: *86-22-2350-6800 \\ Fax: *86-22-2350-9272 \\ duck@santafe.edu
}

\begin{abstract}
In this paper we study abstract shapes of $k$-noncrossing, $\sigma$-canonical RNA pseudoknot structures. We consider $\mid \mathrm{V}_{k^{-}}^{1}$ and $\mid \mathrm{V}_{k}^{5}$-shapes, which represent a generalization of the abstract $\pi^{\prime}$ - and $\pi$-shapes of RNA secondary structures introduced by Giegerich et al. [4]. Using a novel approach we compute the generating functions of $\mathrm{Iv}_{k^{-}}^{1}$ and $I \mathrm{v}_{k}^{5}$-shapes as well as the generating functions of all $\mathrm{Iv}_{k^{-}}^{1}$ and $\mathrm{Iv}_{k}^{5}$-shapes induced by all $k$-noncrossing, $\sigma$-canonical RNA structures for fixed $n$. By means of singularity analysis of the generating functions, we derive explicit asymptotic expressions.
\end{abstract}

Key words:

$k$-noncrossing RNA structure, $\sigma$-canonical, shape, singularity analysis, generating function, core

\section{Introduction}

Pseudoknots have long been known as important structural elements [33], see Fig. 1. They represent cross-serial interactions between RNA nucleotides and are an important functionally in tRNAs, RNaseP [15], telomerase RNA [25], and ribosomal RNAs [14]. Pseudoknots in plant virus RNAs mimic tRNA structures, and in vitro selection experiments have produced pseudoknotted RNA families that bind to the HIV-1 reverse transcriptase [27]. Import general mechanism, such as ribosomal frame shifting, are dependent upon pseudoknots [1]. 

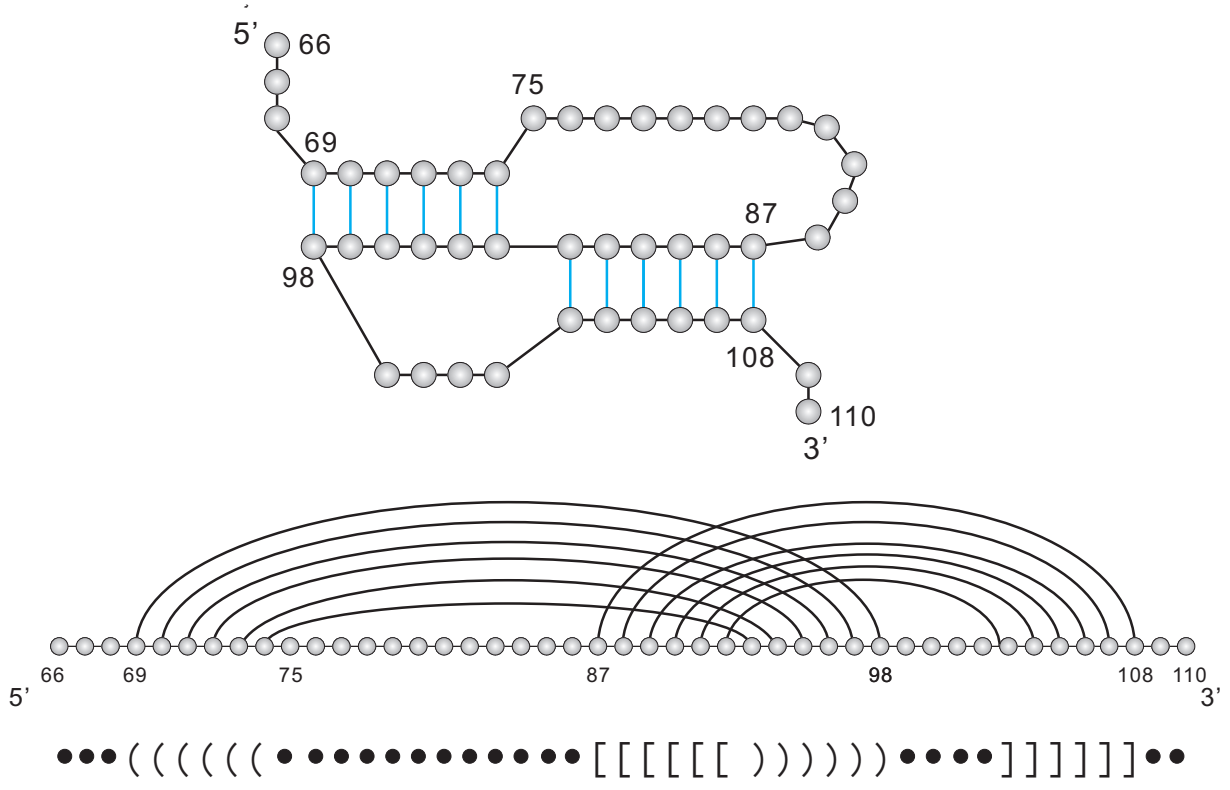

Figure 1: The pseudoknot structure of the PrP-encoding mRNA.

Despite their biological importance, pseudoknots are typically excluded from large-scale computational studies. Although the problem has attracted considerable attention in the last decade, and several software tools [8, 23] have become available, the required resources have remained prohibitive for applications beyond individual molecules.

An RNA molecule is a sequence of the four nucleotides $\mathbf{A}, \mathbf{G}, \mathbf{U}$ and $\mathbf{C}$ together with the Watson-Crick (A-U, G-C) and U-G base pairing rules. The sequence of bases is called the primary structure of the RNA molecule. Two bases in the primary structure which are not adjacent may form hydrogen bonds following the Watson-Crick base pairing rules. Three decades ago Waterman et al. [13, 22, 31] analyzed RNA secondary structures. Secondary structures are coarse grained RNA contact structures. They can be represented as diagrams, planar graphs as well as Motzkin-paths, see Fig. 2. Diagrams are labeled graphs over the vertex set $[n]=\{1, \ldots, n\}$ with vertex degrees $\leq 1$, represented by drawing its vertices on a horizontal line and its $\operatorname{arcs}(i, j)(i<j)$, in the upper half-plane, see Fig. 2 and Fig. 3 . Here, vertices and arcs correspond to the nucleotides $\mathbf{A}, \mathbf{G}, \mathbf{U}$ and $\mathbf{C}$ and Watson-Crick (A-U, G-C) and (U-G) base pairs, respectively. In a diagram two arcs $\left(i_{1}, j_{1}\right)$ and $\left(i_{2}, j_{2}\right)$ are called crossing if $i_{1}<i_{2}<j_{1}<j_{2}$ 

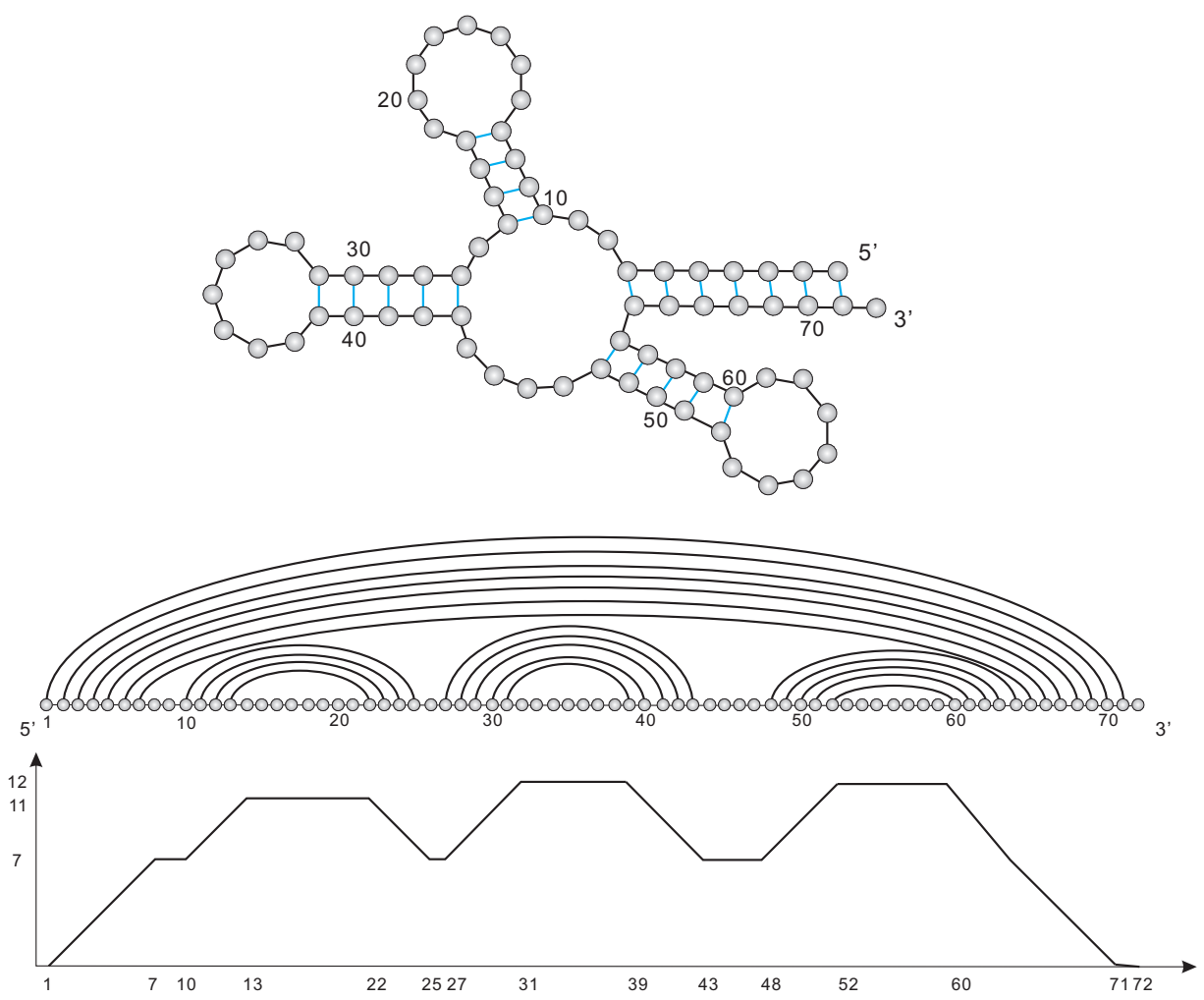

Figure 2: The Sprinzl tRNA RD7550 secondary structure represented as a planar graph (top), 2-noncrossing diagram (middle) and Motzkin-path (bottom), where up/down/horizontal-steps correspond to start/end/unpaired vertices, respectively.

holds. Accordingly, a $k$-crossing is a sequence of $\operatorname{arcs}\left(i_{1}, j_{1}\right), \ldots,\left(i_{k}, j_{k}\right)$ such that $i_{1}<i_{2}<\cdots<i_{k}<j_{1}<j_{2}<\cdots<j_{k}$, see Fig. 3. We call diagrams containing at most $(k-1)$-crossings, $k$-noncrossing diagrams $(k$-noncrossing partial matchings).

An important observation in this context is that RNA secondary structures have no crossings in their diagram representation, see Fig. 3 (1.h.s.) and Fig. 2, and are therefore 2-noncrossing diagrams. The length of an arc $(i, j)$ is given by $j-i$, characterizing the minimal length of a hairpin loop. A stack of length $\sigma$ is a sequence of "parallel" arcs of the form

$$
((i, j),(i+1, j-1), \ldots,(i+(\sigma-1), j-(\sigma-1))) .
$$

In the context of minimum-free energy pseudoknot structures [8] a minimum stack length $\sigma$ or either two or three is stipulated. We remark that RNA sec- 
ondary structures are 2-noncrossing, 2-canonical diagrams, whose numbers are asymptotically given by [6]

$$
S_{2,2}(n) \sim c n^{-3 / 2} 1.96798^{n}, \quad c>0 .
$$

We call an arc of length one a 1 -arc. A $k$-noncrossing, $\sigma$-canonical RNA structure is a $k$-noncrossing diagram without 1 -arcs, having a minimum stack-size of $\sigma$.
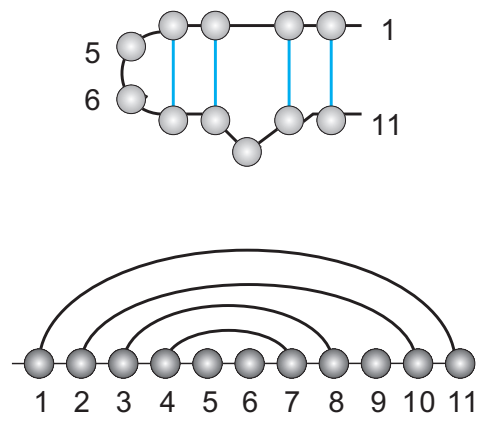
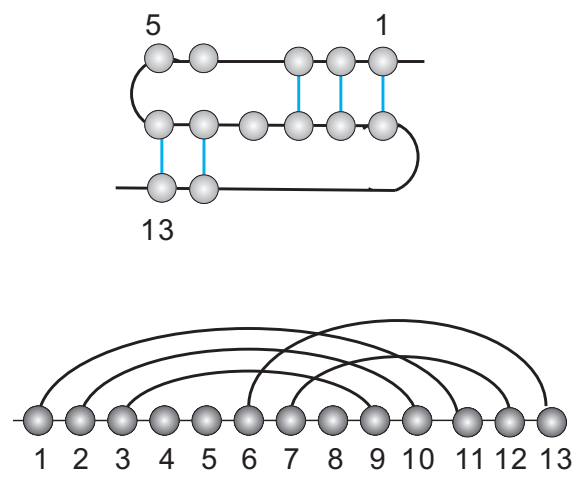

Figure 3: A 2-noncrossing, 2-canonical RNA structure (left) and a 3-noncrossing, 2-canonical RNA structure (right).

The efficient minimum free energy (mfe) folding of secondary structures is a consequence of the following relation of the numbers of RNA secondary structures over $n$ nucleotides, $S_{2}(n),[31$ ]

$$
S_{2}(n)=S_{2}(n-1)+\sum_{j=0}^{n-2} S_{2}(n-2-j) S_{2}(j),
$$

where $S_{2}(n)=1$ for $0 \leq n \leq 2$. Accordingly, RNA secondary structures satisfy a constructive recursion. As mentioned above, this relation is the key for deriving the fundamental DP-recursions used for the polynomial time folding of secondary structures [7, 22] and has therefore profound algorithmic implications. In addition, eq. (3) is of central importance for the analysis of abstract shapes [21]. In addition, for a given RNA sequence, we have not only one but an ensemble of structures, quantified via the partition function generated by the (Boltzman weighted) probability space of all structures [19]. In view of the fact that the number of the mfe and suboptimal foldings 
of an RNA sequence is large, Giegerich et al. [4] introduced the notion of abstract shapes of secondary structures. Two particularly important shape levels are the important level-1 $\left(\pi^{\prime}-\right)$ and level-5 $(\pi-)$ shapes were studied in [4]. In [28], the authors compute the probability of a shape by means of the partition function, where the probability of a shape is the induced probability of all the structures inducing it.

The problem with pseudoknotted structures is, that they do not satisfy a recursion of the type of eq. (3), rendering the $a b$ initio folding into mfe configurations [8, 17] as well as the derivation of any other properties a nontrivial task. Here, we generalize the $\pi^{\prime}$ - and $\pi$-shapes of [4], by introducing $\mathrm{IV}_{k^{-}}^{1}$ and $\mathrm{IV}_{k^{5}}^{5}$-shapes, see Fig. 4. Our results are not new in case of $k=2$,

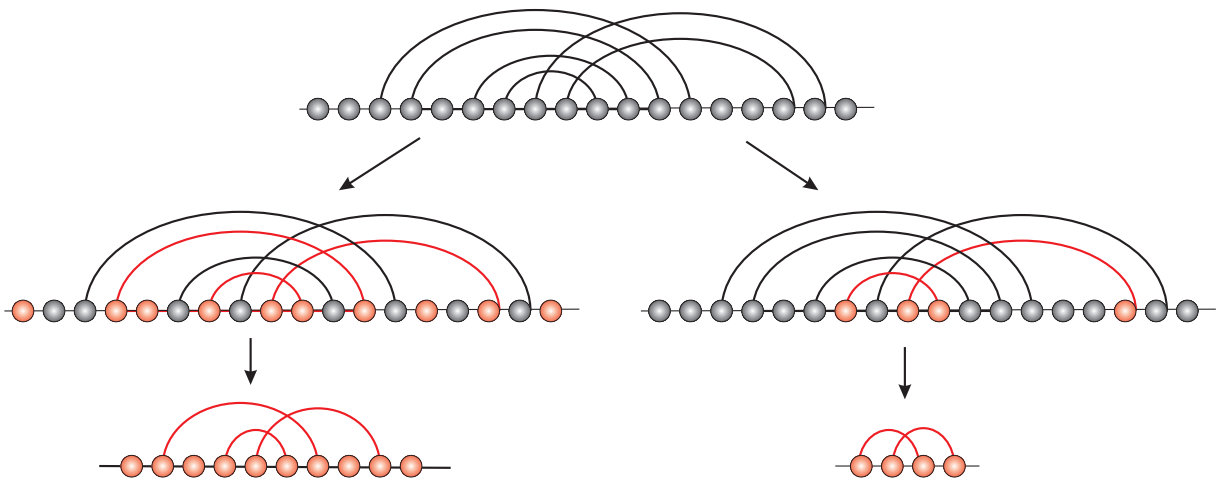

Figure 4: $I v_{k}^{1}$ - and $I v_{k}^{5}$-shapes: a 3-noncrossing, 2-canonical RNA structure (top), its $I v_{3}^{1}$-shape (bottom left) and its $\operatorname{Iv}_{3}^{5}$-shape (bottom right).

since we have $\mid v_{2}^{1}=\pi^{\prime}$ and $I_{2}^{5}=\pi$. In two beautiful papers [16, 21] $\pi^{\prime}$ and $\pi$-shapes have been analyzed. The results of [16, 21] explicitly make use of the constructive recurrence relation given in eq. (3). Their approach can consequently not be generalized to RNA pseudoknot structures, as the latter are genuinely nonrecursive. Our framework therefore identifies the combinatorial "heart" of the results of [16, 21] and provides a new approach avoiding any notion of grammar or recursiveness. The key idea behind the construction of $\mathrm{Iv}_{k^{-}}^{1}$ and $\mathrm{Iv}_{k^{5}}^{5}$-shapes is a projection onto so called $k$-noncrossing core-structures [11].

The paper is organized as follows: after introducing all necessary background we give a detailed computation of the generating functions and study their singularities. We derive simple asymptotic expressions for the numbers of $\mid v_{k}^{1}$ - and $\mid v_{k}^{5}$-shapes as well as the numbers of theses shapes, induced by 
$k$-noncrossing, $\sigma$-canonical RNA structures of fixed length $n$. Finally we put our results into context.

\section{Some basic facts}

Let $f_{k}(n, \ell)$ denote the number of $k$-noncrossing diagrams on $n$ vertices having exactly $\ell$ isolated vertices. A diagram without isolated points is called a matching. The exponential generating function of $k$-noncrossing matchings satisfies the following identity [2, 5, 9]

$$
\sum_{n \geq 0} f_{k}(2 n, 0) \cdot \frac{z^{2 n}}{(2 n) !}=\left.\operatorname{det}\left[I_{i-j}(2 z)-I_{i+j}(2 z)\right]\right|_{i, j=1} ^{k-1}
$$

where $I_{r}(2 z)=\sum_{j \geq 0} \frac{z^{2 j+r}}{j !(j+r) !}$ is the hyperbolic Bessel function of the first kind of order $r$. Eq. (44) allows to conclude that the ordinary generating function

$$
\mathbf{F}_{k}(z)=\sum_{n \geq 0} f_{k}(2 n, 0) z^{n}
$$

is $D$-finite [24], i.e. there exists some $e \in \mathbb{N}$ such that

$$
q_{0, k}(z) \frac{d^{e}}{d z^{e}} \mathbf{F}_{k}(z)+q_{1, k}(z) \frac{d^{e-1}}{d z^{e-1}} \mathbf{F}_{k}(z)+\cdots+q_{e, k}(z) \mathbf{F}_{k}(z)=0,
$$

where $q_{j, k}(z)$ are polynomials. Since $I_{r}(2 z)$ is $D$-finite by its definition and $D$-finite power series are algebraic closed [24]. The key point is that any singularity of $\mathbf{F}_{k}(z)$ is contained in the set of roots of $q_{0, k}(z)$ [24], which we denote by $R_{k}$. For $2 \leq k \leq 9$, we give the polynomials $q_{0, k}(z)$ and their roots in Table 1 .

In [12] we showed that for arbitrary $k$

$$
f_{k}(2 n, 0) \sim \widetilde{c}_{k} n^{-\left((k-1)^{2}+(k-1) / 2\right)}(2(k-1))^{2 n}, \quad \widetilde{c}_{k}>0 .
$$

in accordance with the fact that $\mathbf{F}_{k}(z)$ has the unique dominant singularity $\rho_{k}^{2}$, where $\rho_{k}=1 /(2 k-2)$.

Let $\mathcal{T}_{k, \sigma}(n)$ denote the set of $k$-noncrossing, $\sigma$-canonical RNA structures of length $n$ and let $\mathrm{T}_{k, \sigma}(n)$ denote their number. $\mathcal{T}_{k, \sigma}(n)$ can be identified with the set of $k$-noncrossing RNA structures with each stack size $\geq \sigma$. Furthermore, let $\mathcal{T}_{k, \sigma}(n, h)$ denote the set of $k$-noncrossing, $\sigma$-canonical RNA 


\begin{tabular}{lll}
\hline$k$ & $q_{0, k}(z)$ & $R_{k}$ \\
\hline 2 & $(4 z-1) z$ & $\left\{\frac{1}{4}\right\}$ \\
3 & $(16 z-1) z^{2}$ & $\left\{\frac{1}{16}\right\}$ \\
4 & $\left(144 z^{2}-40 z+1\right) z^{3}$ & $\left\{\frac{1}{4}, \frac{1}{36}\right\}$ \\
5 & $\left(1024 z^{2}-80 z+1\right) z^{4}$ & $\left\{\frac{1}{16}, \frac{1}{64}\right\}$ \\
6 & $\left(14400 z^{3}-4144 z^{2}+140 z-1\right) z^{5}$ & $\left\{\frac{1}{4}, \frac{1}{36}, \frac{1}{100}\right\}$ \\
7 & $\left(147456 z^{3}-12544 z^{2}+224 z-1\right) z^{6}$ & $\left\{\left\{\frac{1}{16}, \frac{1}{64}, \frac{1}{14}\right\}\right.$ \\
8 & $\left(2822400 z^{4}-826624 z^{3}+31584 z^{2}-336 z+1\right) z^{7}$ & $\left\{\frac{1}{4}, \frac{1}{66}, \frac{1}{100}, \frac{1}{196}\right\}$ \\
9 & $\left(37748736 z^{4}-3358720 z^{3}+69888 z^{2}-480 z+1\right) z^{8}$ & $\left\{\frac{1}{16}, \frac{1}{64}, \frac{1}{144}, \frac{1}{256}\right\}$ \\
\hline
\end{tabular}

Table 1: We present the polynomials $q_{0, k}(z)$ and their nonzero roots obtained by the MAPLE package GFUN.

structures of length $n$ with $h$ arcs, and set $\mathrm{T}_{k, \sigma}(n, h)=\left|\mathcal{T}_{k, \sigma}(n, h)\right|$. The bivariate generating function of $\mathrm{T}_{k, 1}(n, h)(k \geq 2)$ has been computed in [10]

$$
\sum_{n \geq 0} \sum_{h=0}^{\left\lfloor\frac{n}{2}\right\rfloor} \mathrm{T}_{k, 1}(n, h) v^{h} y^{n}=\frac{1}{v y^{2}-y+1} \mathbf{F}_{k}\left(\frac{v y^{2}}{\left(v y^{2}-y+1\right)^{2}}\right)
$$

and the generating function for $k$-noncrossing, $\sigma$-canonical RNA structures is given by [11]

$$
\sum_{n \geq 0} \mathrm{~T}_{k, \sigma}(n) y^{n}=\frac{1}{u_{0} y^{2}-y+1} \mathbf{F}_{k}\left(\left(\frac{\sqrt{u_{0}} y}{u_{0} y^{2}-y+1}\right)^{2}\right),
$$

where $u_{0}=\frac{\left(y^{2}\right)^{\sigma-1}}{\left(y^{2}\right)^{\sigma}-y^{2}+1}$.

According to Pringsheim's Theorem [3, 26], each power series $f(z)=$ $\sum_{n \geq 0} a_{n} z^{n}$ with nonnegative coefficients and a radius of convergence $R>0$ has a positive real dominant singularity at $z=R$. This singularity plays a key role for the asymptotics of the coefficients. The class of theorems that deal with such deductions are called transfer-theorems [3]. One key ingredient in this framework is a specific domain in which the functions in question are analytic, which is "slightly" bigger than their respective radius of convergence. It is tailored for extracting the coefficients via Cauchy's integral formula. Details on the method can be found in [3, 24]. In case 
of $D$-finite functions we have analytic continuation in any simply connected domain containing zero [29] and all prerequisites of singularity analysis are met. We use the notation

$$
\{f(z)=O(g(z)) \text { as } z \rightarrow \rho\} \quad \Longleftrightarrow \quad\left\{\frac{f(z)}{g(z)} \text { is bounded as } z \rightarrow \rho\right\} .
$$

Let $\left[z^{n}\right] f(z)$ denote the $n$-th coefficient of the power series $f(z)$ at $z=0$.

Theorem 2.1. [3] Let $f(z), g(z)$ be D-finite functions with unique dominant singularity $\rho$ and suppose

$$
f(z)=O(g(z)) \text { as } z \rightarrow \rho .
$$

Then we have

$$
\left[z^{n}\right] f(z)=C\left(1-O\left(\frac{1}{n}\right)\right)\left[z^{n}\right] g(z)
$$

where $C$ is a constant.

Theorem 2.1 implies the following result, tailored for our functional equations. It is a particular instance of the supercritical paradigm, where we have the following situation: we are given a $D$-finite function, $f(z)$ and an algebraic function $g(u)$ satisfying $g(0)=0$. Furthermore we suppose that $f(g(u))$ has the unique real valued dominant singularity $\gamma$ and $g$ is regular in a disc with radius slightly larger than $\gamma$. The supercritical paradigm then stipulates that the subexponential factors of $f(g(u))$ at $u=0$ coincide with those of $f(z)$.

Proposition 1. Suppose $\vartheta_{\sigma}(z)$ is an algebraic function, analytic for $|z|<\delta$ and satisfies $\vartheta_{\sigma}(0)=0$. Suppose further $\gamma_{k, \sigma}<\delta$ is the real unique dominant singularity of $\mathbf{F}_{k}\left(\vartheta_{\sigma}(z)\right)$ and satisfies $\vartheta_{\sigma}\left(\gamma_{k, \sigma}\right)=\rho_{k}^{2}$. Then

$$
\left[z^{n}\right] \mathbf{F}_{k}\left(\vartheta_{\sigma}(z)\right) \sim c_{k} n^{-\left((k-1)^{2}+(k-1) / 2\right)}\left(\gamma_{k, \sigma}^{-1}\right)^{n} \text {. }
$$

Let $\mathcal{G}_{k}(n, m)$ denote the set of the $k$-noncrossing matchings of length $2 n$ with $m$ 1-arcs. In our first lemma, we will compute the bivariate generating function of $g_{k}(n, m)$, i.e. the number of $k$-noncrossing matchings of length $2 n$ with exactly $m$ 1-arcs. 
Lemma 2.2. Suppose $k, n, m \in \mathbb{N}, k \geq 2,0 \leq m \leq n$. Then $g_{k}(n, m)$ satisfies the recursion

$$
(m+1) g_{k}(n+1, m+1)=(m+1) g_{k}(n, m+1)+(2 n+1-m) g_{k}(n, m) .
$$

Furthermore, the generating function $\mathbf{G}_{k}(x, y)=\sum_{n \geq 0} \sum_{m=0}^{n} g_{k}(n, m) x^{n} y^{m}$ is given by

$$
\mathbf{G}_{k}(x, y)=\frac{1}{x+1-y x} \mathbf{F}_{k}\left(\frac{x}{(x+1-y x)^{2}}\right) .
$$

Proof. Choose a $k$-noncrossing matching $\delta \in \mathcal{G}_{k}(n+1, m+1)$ and label one 1 -arc. We have $(m+1) g_{k}(n+1, m+1)$ different such labeled $k$-noncrossing matchings. On the other hand, in order to obtain such a labeled matching, we can also insert one labeled 1 -arc in a $k$-noncrossing matching $\delta^{\prime} \in \mathcal{G}_{k}(n, m+1)$. In this case, we can only put it inside one original 1-arc in $\delta^{\prime}$ in order to preserve the number of the 1-arcs. We may also insert a labeled 1-arc in a $k$-noncrossing matching $\delta^{\prime \prime} \in \mathcal{G}_{k}(n, m)$. In this case, we can only insert the 1-arc between two vertices not forming a 1-arc. Therefore, we arrive at $(m+1) g_{k}(n, m+1)+(2 n+1-m) g_{k}(n, m)$ different such labeled matchings and

$$
(m+1) g_{k}(n+1, m+1)=(m+1) g_{k}(n, m+1)+(2 n+1-m) g_{k}(n, m) .
$$

This recursion implies the following partial differential equation for the generating function

$$
x^{-1} \frac{\partial \mathbf{G}_{k}(x, y)}{\partial y}=\frac{\partial \mathbf{G}_{k}(x, y)}{\partial y}+2 x \frac{\partial \mathbf{G}_{k}(x, y)}{\partial x}+\mathbf{G}_{k}(x, y)-y \frac{\partial \mathbf{G}_{k}(x, y)}{\partial y},
$$

whose general solution is given by

$$
\mathbf{G}_{k}(x, y)=\frac{F\left(\frac{y x-1-x}{\sqrt{x}}\right)}{\sqrt{x}}
$$

where $F(z)$ is an arbitrary function. By definition, we have $\sum_{m=0}^{n} g_{k}(n, m)=$ $f_{k}(2 n, 0)$ and

$$
\mathbf{G}_{k}(x, 1)=\sum_{n \geq 0} f_{k}(2 n, 0) x^{n} .
$$

Using eq. (16) and eq. (18) we derive

$$
\mathbf{G}_{k}(x, y)=\frac{1}{x+1-y x} \sum_{n \geq 0} f_{k}(2 n, 0)\left(\frac{x}{(x+1-y x)^{2}}\right)^{n},
$$

whence the lemma. 


\section{Combinatorics of $\mathbf{I v}_{k}^{5}$-shapes}

We now show how to derive the $1 \mathbf{v}_{k}^{5}$-shape of a given $k$-noncrossing, $\sigma$ canonical RNA structures. This construction is based on the notion of $k$ noncrossing cores [11]. A $k$-noncrossing core is a $k$-noncrossing RNA structure in which each stack has size exactly one. The cores of a $k$-noncrossing, $\sigma$-canonical RNA structure, $\delta$, denoted by $c(\delta)$ is obtained in two steps: first we map arcs and isolated vertices as follows:

$$
\forall \ell \geq \sigma-1 ; \quad((i-\ell, j+\ell), \ldots,(i, j)) \mapsto(i, j) \text { and } j \mapsto j \text { if } j \text { is isolated (20) }
$$

and second we relabel the vertices of the resulting diagram from left to right in increasing order, see Fig. 5 . We are now in position to define $\mathrm{Iv}_{k}^{5}$-shapes.

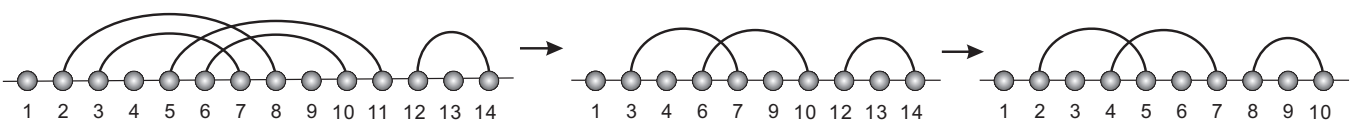

Figure 5: A 3-noncrossing core structure is obtained from a 3-noncrossing, 1canonical RNA structure in two steps.

Definition 1. (IV ${ }_{k}^{5}$-shape) Given a $k$-noncrossing, $\sigma$-canonical RNA structure $\delta$, its $\mathbf{I V}_{k}^{5}$-shape, $\mathbf{I v}_{k}^{5}(\delta)$, is obtained by first removing all isolated vertices and second apply the core-map $c$.

Alternatively the $\mathrm{IV}_{k}^{5}$-shape can also be derived as follows: we first project into the core $c(\delta)$, second, we remove all isolated vertices and third we apply the core-map $c$ again, see Fig, 6 . The second step is a projection from $k$ -

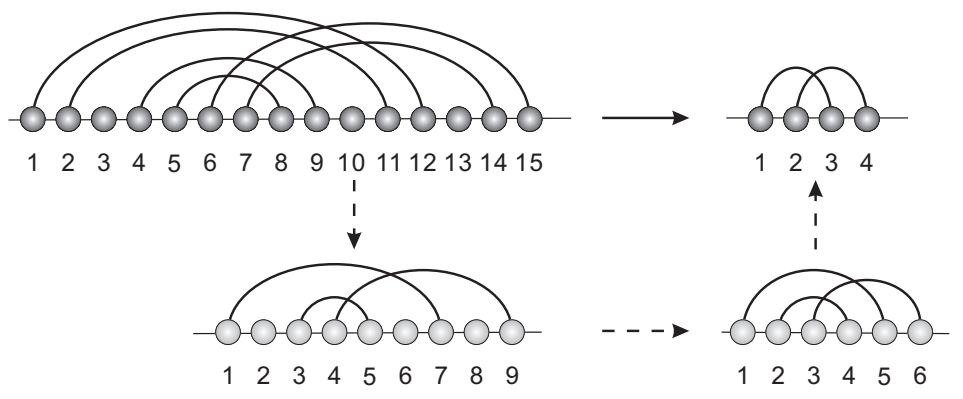

Figure 6: Two methods for generating the $\mathrm{IV}_{3}^{5}$-shape. A 3-noncrossing, 2-canonical RNA structure (top-left) is mapped in two ways into its $\mathrm{Iv}_{3}^{5}$-shape (top-right). 
noncrossing cores to $k$-noncrossing matchings and surjective, since for each $k$-noncrossing matching $\alpha$, we can obtain a core structure by inserting isolated vertices between any two arcs contained in some stack. By construction, $\operatorname{lv}_{k}^{5}$ shapes do not preserve stack-lengths, interior loops and unpaired regions.

Let $\mathcal{I}_{k}(n, m)\left(i_{k}(n, m)\right)$ denote the set (number) of the $\mathbf{I v}_{k}^{5}$-shapes of length $2 n$ with $m$ 1-arcs and

$$
\mathbf{I}_{k}(z, u)=\sum_{n \geq 0} \sum_{m=0}^{n} i_{k}(n, m) z^{n} u^{m}
$$

be the bivariate generating function. Furthermore, let $i_{k}(n)$ denote the number of the $\mathrm{Iv}_{k}^{5}$-shapes of length $2 n$ with generating function

$$
\mathbf{I}_{k}(z)=\sum_{n \geq 0} i_{k}(n) z^{n}
$$

Since any $\operatorname{lv}_{k}^{5}$-shape is in particular the core of some $k$-noncrossing matching, Lemma 2.2 allows us to establish a relation between the bivariate generating function of $i_{k}(n, m)$ and the generating function of $\mathbf{F}_{k}(z)$.

Theorem 3.1. Let $k, n, m$ be natural numbers where $k \geq 2$, then the following assertions hold

(a) the generating functions $\mathbf{I}_{k}(z, u)$ and $\mathbf{I}_{k}(z)$ satisfy

$$
\begin{aligned}
\mathbf{I}_{k}(z, u) & =\frac{1+z}{1+2 z-z u} \mathbf{F}_{k}\left(\frac{z(1+z)}{(1+2 z-z u)^{2}}\right) \\
\mathbf{I}_{k}(z) & =\mathbf{F}_{k}\left(\frac{z}{1+z}\right)
\end{aligned}
$$

(b) for $2 \leq k \leq 9$, the number of $\mid \mathrm{v}_{k}^{5}$-shapes of length $2 n$ is asymptotically given by

$$
i_{k}(n) \sim c_{k} n^{-\left((k-1)^{2}+(k-1) / 2\right)}\left(\mu_{k}^{-1}\right)^{n},
$$

where $\mu_{k}$ is the unique minimum positive real solution of $\frac{z}{1+z}=\rho_{k}^{2}$ and $c_{k}$ is some positive constant.

Proof. We first prove (a). For this purpose we define a map between $k$-noncrossing matchings with $m 1$-arcs and $\operatorname{lv}_{k}^{5}$-shapes

$$
g: \mathcal{G}_{k}(n, m) \rightarrow \bigcup_{0 \leq b \leq n-m}\left[\mathcal{I}_{k}(n-b, m) \times\left\{\left(a_{j}\right)_{1 \leq j \leq n-b} \mid \sum_{j=1}^{n-b} a_{j}=b, a_{j} \geq 0\right\}\right]
$$


where $n \geq 1$. Here, for every $\delta \in \mathcal{G}_{k}(n, m)$, we have $g(\delta)=\left(c(\delta),\left(a_{j}\right)_{1 \leq j \leq n-b}\right)$, where $c(\delta)$ is the core structure of $\delta$ obtained according to eq. (20) and where $\left(a_{j}\right)_{1 \leq j \leq n-b}$ keeps track of the deleted arcs. It is straightforward to check that the map $g$ is well defined, since all the 1-arcs of $c(\delta)$ are just the 1-arcs of $\delta$. By construction, $g$ is a bijection and we have

$$
\left|\left\{\left(a_{j}\right)_{1 \leq j \leq n-b} \mid \sum_{j=1}^{n-b} a_{j}=b, a_{j} \geq 0\right\}\right|=\left(\begin{array}{c}
n-1 \\
b
\end{array}\right) .
$$

Then we derive

$$
g_{k}(n, m)=\sum_{b=0}^{n-m}\left(\begin{array}{c}
n-1 \\
b
\end{array}\right) i_{k}(n-b, m), \quad \text { for } n \geq 1,
$$

which implies

$$
\sum_{n \geq 0} \sum_{m=0}^{n} g_{k}(n, m) x^{n} y^{m}=\sum_{n \geq 1} \sum_{m=0}^{n} \sum_{b=0}^{n-m}\left(\begin{array}{c}
n-1 \\
b
\end{array}\right) i_{k}(n-b, m) x^{n} y^{m}+1 .
$$

We next observe

$\sum_{n \geq 1} \sum_{m=0}^{n} \sum_{b=0}^{n-m}\left(\begin{array}{c}n-1 \\ b\end{array}\right) i_{k}(n-b, m) x^{n} y^{m}=\sum_{b \geq 0} \sum_{m \geq 0} \sum_{n \geq n_{0}}\left(\begin{array}{c}n-1 \\ b\end{array}\right) i_{k}(n-b, m) x^{n} y^{m}$,

where $n_{0}=\max \{m+b, 1\}$ and setting $s=n-b$,

$\sum_{n \geq 1} \sum_{m=0}^{n} \sum_{b=0}^{n-m}\left(\begin{array}{c}n-1 \\ b\end{array}\right) i_{k}(n-b, m) x^{n} y^{m}=\sum_{b \geq 0} \sum_{m \geq 0} \sum_{s \geq s_{0}}\left(\begin{array}{c}s+b-1 \\ b\end{array}\right) i_{k}(s, m) x^{s+b} y^{m}$

where $s_{0}=\max \{m, 1\}$. In view of

$$
\sum_{b \geq 0}\left(\begin{array}{c}
s+b-1 \\
b
\end{array}\right) x^{b}=\frac{1}{(1-x)^{s}}
$$

and interchanging the terms of summation, we derive

$$
\sum_{n \geq 1} \sum_{m=0}^{n} \sum_{b=0}^{n-m}\left(\begin{array}{c}
n-1 \\
b
\end{array}\right) i_{k}(n-b, m) x^{n} y^{m}=\sum_{s \geq 1} \sum_{m=0}^{s} i_{k}(s, m)\left(\frac{x}{1-x}\right)^{s} y^{m}
$$


and arrive at

$$
\sum_{n \geq 0} \sum_{m=0}^{n} g_{k}(n, m) x^{n} y^{m}=\sum_{n \geq 0} \sum_{m=0}^{n} i_{k}(n, m)\left(\frac{x}{1-x}\right)^{n} y^{m} .
$$

According to Lemma 2.2, we have

$$
\sum_{n \geq 0} \sum_{m=0}^{n} g_{k}(n, m) x^{n} y^{m}=\frac{1}{x+1-y x} \sum_{n \geq 0} f_{k}(2 n, 0)\left(\frac{x}{(x+1-y x)^{2}}\right)^{n},
$$

setting $z=\frac{x}{1-x}$ and $u=y$,

$$
\sum_{n \geq 0} \sum_{m=0}^{n} i_{k}(n, m) z^{n} u^{m}=\frac{1+z}{1+2 z-z u} \sum_{n \geq 0} f_{k}(2 n, 0)\left(\frac{z(1+z)}{(1+2 z-z u)^{2}}\right)^{n} .
$$

In particular, setting $u=1$, we derive

$$
\sum_{n \geq 0} i_{k}(n) z^{n}=\sum_{n \geq 0} f_{k}(2 n, 0)\left(\frac{z}{1+z}\right)^{n}
$$

whence (a) follows.

Assertion (b) is a direct consequence of the supercritical paradigm, see Proposition 1. As mentioned before, the ordinary generating function $\mathbf{F}_{k}(z)=$ $\sum_{n \geq 0} f_{k}(2 n, 0) z^{n}$ is $D$-finite [24] and the inner function $\vartheta(z)=\frac{z}{1+z}$ is algebraic, satisfies $\vartheta(0)=0$ and is analytic for $|z|<1$. By direct calculation, using the fact that all singularities of $\mathbf{F}_{k}(z)$ are contained within the set of zeros of $q_{0, k}(z)$, see Tab. 1, we can then verify that $\mathbf{F}_{k}(\vartheta(z))$ has the unique dominant real singularity $\mu_{k}<1$ satisfying $\vartheta\left(\mu_{k}\right)=\rho_{k}^{2}$ for $2 \leq k \leq 9$. In view of $f_{k}(2 n, 0) \sim \widetilde{c}_{k} n^{-\left((k-1)^{2}+(k-1) / 2\right)}(2(k-1))^{2 n}$, Proposition 1 guarantees eq. (25)

$$
i_{k}(n) \sim c_{k} n^{-\left((k-1)^{2}+(k-1) / 2\right)}\left(\mu_{k}^{-1}\right)^{n} .
$$

This proves (b) completing the proof of the theorem.

We next studying the number of $\mathrm{Iv}_{k}^{5}$-shapes induced by $k$-noncrossing, $\sigma$-canonical RNA structures of fixed length $n, \operatorname{Iv}_{k, \sigma}^{5}(n)$, setting

$$
\mathbf{L v}_{k, \sigma}^{5}(x)=\sum_{n \geq 0} \mid \mathbf{v}_{k, \sigma}^{5}(n) x^{n}
$$


Theorem 3.2. Let $k, \sigma \in \mathbb{N}$, where $k \geq 2$. Then the following assertions hold

(a) the generating function $\mathbf{L v}_{k, \sigma}^{5}(x)$ is given by

$$
\mathbf{L v}_{k, \sigma}^{5}(x)=\frac{\left(1+x^{2 \sigma}\right)}{(1-x)\left(1+2 x^{2 \sigma}-x^{2 \sigma+1}\right)} \mathbf{F}_{k}\left(\frac{x^{2 \sigma}\left(1+x^{2 \sigma}\right)}{\left(1+2 x^{2 \sigma}-x^{2 \sigma+1}\right)^{2}}\right) .
$$

(b) for $2 \leq k \leq 9$ and $1 \leq \sigma \leq 10$

$$
\operatorname{Iv}_{k, \sigma}^{5}(n) \sim c_{k, \sigma} n^{-\left((k-1)^{2}+(k-1) / 2\right)}\left(\zeta_{k, \sigma}^{-1}\right)^{n},
$$

where $c_{k, \sigma}>0$ and $\zeta_{k, \sigma}$ is the unique minimum positive real solution of

$$
\frac{x^{2 \sigma}\left(1+x^{2 \sigma}\right)}{\left(1+2 x^{2 \sigma}-x^{2 \sigma+1}\right)^{2}}=\rho_{k}^{2}
$$

\begin{tabular}{cccccccc}
\hline$\sigma / k$ & 2 & 3 & 4 & 5 & 6 & 7 & 8 \\
\hline 1 & 1.51243 & 3.67528 & 5.77291 & 7.82581 & 9.85873 & 11.88118 & 13.89746 \\
2 & 1.26585 & 1.93496 & 2.41152 & 2.80275 & 3.14338 & 3.44943 & 3.72983 \\
3 & 1.17928 & 1.55752 & 1.80082 & 1.98945 & 2.14693 & 2.28376 & 2.40567 \\
\hline
\end{tabular}

Table 2: The exponential growth rates $\zeta_{k, \sigma}^{-1}$ of $\operatorname{Iv}_{k}^{5}$-shapes induced by $k$-noncrossing, $\sigma$-canonical RNA structures of length $n$.

Proof. In order to proof of (a) we observe that we can always inflate a structure by adding arcs to stacks or isolated vertices without changing its $\mathbf{I} \mathbf{v}_{k}^{5}$-shape. In fact, for any given $\mathrm{Iv}_{k}^{5}$-shape, $\beta$, adding the minimal number of arcs to each stack such that every stack has $\sigma$ arcs, and inserting one isolated vertex in any 1 -arc, we derive a $k$-noncrossing, $\sigma$-canonical structure having arc-length $\geq 2$, of minimal length. We can therefore derive $\mathbf{L v}_{k, \sigma}^{5}(x)$, see eq.(27), from the bivariate generating function $\mathbf{I}_{k}(z, u)$ as follows

$$
\mathbf{L v}_{k, \sigma}^{5}(x)=\sum_{n \geq 0} \sum_{s=0}^{\left\lfloor\frac{n}{2 \sigma}\right\rfloor} \sum_{m=0}^{\min \{s, n-2 \sigma s\}} i_{k}(s, m) x^{n}=\sum_{s \geq 0} \sum_{m=0}^{s} \sum_{n \geq 2 \sigma s+m} i_{k}(s, m) x^{n},
$$


whence

$$
\mathbf{L v}_{k, \sigma}^{5}(x)=\frac{1}{1-x} \sum_{s \geq 0} \sum_{m=0}^{s} i_{k}(s, m) x^{2 \sigma s+m}
$$

and in view of eq. (23), $\mathbf{I}_{k}(z, u)=\frac{1+z}{1+2 z-z u} \mathbf{F}_{k}\left(\frac{z(1+z)}{(1+2 z-z u)^{2}}\right)$, we derive

$$
\mathbf{L v}_{k, \sigma}^{5}(x)=\frac{\left(1+x^{2 \sigma}\right)}{(1-x)\left(1+2 x^{2 \sigma}-x^{2 \sigma+1}\right)} \mathbf{F}_{k}\left(\frac{x^{2 \sigma}\left(1+x^{2 \sigma}\right)}{\left(1+2 x^{2 \sigma}-x^{2 \sigma+1}\right)^{2}}\right) .
$$

As for (b), we observe that the factor

$$
\varphi_{\sigma}(x)=\frac{\left(1+x^{2 \sigma}\right)}{(1-x)\left(1+2 x^{2 \sigma}-x^{2 \sigma+1}\right)}
$$

does not induce a dominant singularity of $\mathbf{L v}_{k, \sigma}^{5}(x)$. Therefore all dominant singularities of $\mathbf{L v}_{k, \sigma}^{5}(x)$ stem from $\mathbf{F}_{k}\left(\frac{x^{2 \sigma}\left(1+x^{2 \sigma}\right)}{\left(1+2 x^{2 \sigma}-x^{2 \sigma+1}\right)^{2}}\right)$. Indeed, assume $a$ contrario that there were some dominant singularity of $\mathbf{L v}_{k, \sigma}^{5}(x), \zeta$, that is induced by $\varphi_{\sigma}(x)$. This would imply that $\zeta$ is also a dominant singularity of $\mathbf{F}_{k}\left(\frac{x^{2 \sigma}\left(1+x^{2 \sigma}\right)}{\left(1+2 x^{2 \sigma}-x^{2 \sigma+1}\right)^{2}}\right)$ which immediately leads to a contradiction.

We next verify that for $2 \leq k \leq 9$ and $1 \leq \sigma \leq 10$, the minimum positive real solution of eq. (30), $\zeta_{k, \sigma}$, is the unique dominant singularity of $\mathbf{L v}_{k, \sigma}^{5}(x)$ and Proposition 1 implies

$$
\operatorname{Iv}_{k, \sigma}^{5}(n) \sim c_{k, \sigma} n^{-\left((k-1)^{2}+(k-1) / 2\right)}\left(\zeta_{k, \sigma}^{-1}\right)^{n}
$$

where $c_{k, \sigma}$ is some positive constant and the proof of the theorem is complete.

\section{Combinatorics of $\mathbf{I v}_{k}^{1}$-shapes}

Definition 2. ( $\mathrm{v}_{k}^{1}$-shape) Given a $k$-noncrossing, $\sigma$-canonical RNA structure, $\delta$, its $\operatorname{lv}_{k}^{1}$-shape, $\operatorname{lv}_{k}^{1}(\delta)$, is derived as follows: first we apply the core map, second we replace a segment of isolated vertices by a single isolated vertex and third relabel the vertices of the resulting diagram, see Fig.7.

More formally, a $\operatorname{Iv}_{k}^{1}$-shape is obtained as follows: if we have a maximal sequence of isolated vertices $\left(i, i+1, \ldots, i+\ell^{\prime}\right)$ (i.e. $i-1, i+\ell^{\prime}+1$ are not isolated), then we map $\left(i, i+1, \ldots, i+\ell^{\prime}\right) \mapsto i$ and if $(i, j)$ is a arc, it is mapped identically. 


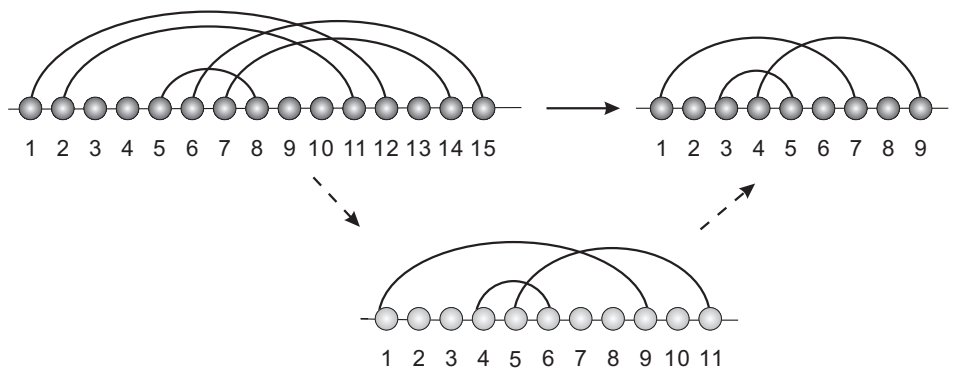

Figure $7: \operatorname{Iv}_{k}^{1}$-shapes via the core map and subsequent identification of unpaired nucleotides: A 3-noncrossing, 1-canonical RNA structure (top-left) is mapped into its $I v_{3}^{1}$-shape (top-right).

Let $\mathcal{C}_{k}(n, h)\left(C_{k}(n, h)\right)$ denote the set (number) of $k$-noncrossing corestructures of length $n$ with exactly $h$-arcs. Let $\mathcal{J}_{k}(n, h)\left(j_{k}(n, h)\right)$ denote the set (number) of $\mathbf{l v}_{k}^{1}$-shapes of length $n$ with $h$-arcs, and let $j_{k}(n)$ be the number of all $\mathrm{Iv}_{k}^{1}$-shapes of length $n$ and set

$$
\mathbf{J}_{k}(z, u)=\sum_{h \geq 0} \sum_{n=2 h}^{4 h+1} j_{k}(n, h) z^{n} u^{h} \text { and } \mathbf{J}_{k}(z)=\sum_{n \geq 0} j_{k}(n) z^{n} .
$$

Theorem 4.1. For $k, n, h \in \mathbb{N}, k \geq 2$, the following assertions hold (a) the generating functions $\mathbf{J}_{k}(z, u)$ and $\mathbf{J}_{k}(z)$ are given by

$$
\begin{aligned}
\mathbf{J}_{k}(z, u) & =\frac{(1+z)\left(1+u z^{2}\right)}{u z^{3}+2 u z^{2}+1} \mathbf{F}_{k}\left(\frac{(1+z)^{2}\left(1+u z^{2}\right) u z^{2}}{\left(u z^{3}+2 u z^{2}+1\right)^{2}}\right) \\
\mathbf{J}_{k}(z) & =\frac{(1+z)\left(1+z^{2}\right)}{z^{3}+2 z^{2}+1} \mathbf{F}_{k}\left(\frac{(1+z)^{2}\left(1+z^{2}\right) z^{2}}{\left(z^{3}+2 z^{2}+1\right)^{2}}\right) .
\end{aligned}
$$

(b) for $2 \leq k \leq 9$, the number of $\operatorname{lv}_{k}^{1}$-shapes of length $n$ satisfies

$$
j_{k}(n) \sim c_{k}^{\prime} n^{-\left((k-1)^{2}+(k-1) / 2\right)}\left(\mu_{k}^{\prime-1}\right)^{n},
$$

where $c_{k}^{\prime}>0$ and $\mu_{k}^{\prime}$ is the unique minimum positive real solution of

$$
\frac{(1+z)^{2}\left(1+z^{2}\right) z^{2}}{\left(z^{3}+2 z^{2}+1\right)^{2}}=\rho_{k}^{2}
$$


Proof. For (a) we consider the map between $k$-noncrossing cores having exactly $h$ arcs and $\mid \mathrm{v}_{k}^{1}$-shapes, for $0 \leq h \leq\left\lfloor\frac{n-1}{2}\right\rfloor$,

$$
\begin{aligned}
& \ell: \mathcal{C}_{k}(n, h) \rightarrow \\
& \bigcup_{b_{0} \leq b \leq n-2 h-1}\left[\mathcal{J}_{k}(n-b, h) \times\left\{\left(e_{j}\right)_{1 \leq j \leq n-2 h-b} \mid \sum_{j=1}^{n-2 h-b} e_{j}=b, e_{j} \geq 0\right\}\right],
\end{aligned}
$$

where $b_{0}=\max \{0, n-4 h-1\}$. For every $\beta \in \mathcal{C}_{k}(n, h),\left(e_{j}\right)_{1 \leq j \leq n-2 h-b}$ keeps track of the multiplicities of the deleted isolated vertices. The map $\ell$ is a (well defined) bijection and

$$
\left|\left\{\left(e_{j}\right)_{1 \leq j \leq n-2 h-b} \mid \sum_{j=1}^{n-2 h-b} e_{j}=b, e_{j} \geq 0\right\}\right|=\left(\begin{array}{c}
n-2 h-1 \\
b
\end{array}\right) .
$$

We arrive at

$$
C_{k}(n, h)=\sum_{b=b_{0}}^{n-2 h-1}\left(\begin{array}{c}
n-2 h-1 \\
b
\end{array}\right) j_{k}(n-b, h), \quad 0 \leq h \leq\left\lfloor\frac{n-1}{2}\right\rfloor .
$$

We compute

$$
\begin{aligned}
\sum_{n \geq 0} \sum_{h=0}^{\left\lfloor\frac{n}{2}\right\rfloor} C_{k}(n, h) w^{h} x^{n}= & \underbrace{\sum_{n \geq 0} \sum_{h>\left\lfloor\frac{n-1}{2}\right\rfloor}^{\left\lfloor\frac{n}{2}\right\rfloor} C_{k}(n, h) w^{h} x^{n}}_{(\mathrm{I})}+ \\
& \underbrace{\sum_{n \geq 0} \sum_{h=0}^{\left\lfloor\frac{n-1}{2}\right\rfloor} \sum_{b=b_{0}}^{n-2 h-1}\left(\begin{array}{c}
n-2 h-1 \\
b
\end{array}\right) j_{k}(n-b, h) w^{h} x^{n}}_{\text {(II) }},
\end{aligned}
$$

and rewrite (II) as

$$
\begin{aligned}
& \sum_{n \geq 0} \sum_{h=0}^{\left\lfloor\frac{n-1}{2}\right\rfloor} \sum_{b=b_{0}}^{n-2 h-1}\left(\begin{array}{c}
n-2 h-1 \\
b
\end{array}\right) j_{k}(n-b, h) w^{h} x^{n} \\
= & \sum_{h \geq 0} \sum_{b \geq 0} \sum_{n=2 h+b+1}^{4 h+b+1} j_{k}(n-b, h)\left(\begin{array}{c}
n-2 h-1 \\
b
\end{array}\right) w^{h} x^{n} .
\end{aligned}
$$


We derive, setting $s=n-b$,

$$
\begin{aligned}
& =\sum_{h \geq 0} \sum_{b \geq 0} \sum_{s=2 h+1}^{4 h+1} j_{k}(s, h)\left(\begin{array}{c}
s+b-2 h-1 \\
b
\end{array}\right) w^{h} x^{s+b} \\
& =\sum_{h \geq 0} \sum_{s=2 h+1}^{4 h+1} j_{k}(s, h)\left(\sum_{b \geq 0}\left(\begin{array}{c}
s+b-2 h-1 \\
b
\end{array}\right) x^{b}\right) w^{h} x^{s} \\
& =\sum_{h \geq 0} \sum_{s=2 h+1}^{4 h+1} j_{k}(s, h)\left(\frac{x}{1-x}\right)^{s}\left((1-x)^{2} w\right)^{h} .
\end{aligned}
$$

In view of $j_{k}(2 h, h)=C_{k}(2 h, h)$, we can interpret (I) as follows

$$
\sum_{n \geq 0} \sum_{h>\left\lfloor\frac{n-1}{2}\right\rfloor}^{\left\lfloor\frac{n}{2}\right\rfloor} C_{k}(n, h) w^{h} x^{n}=\sum_{h \geq 0} j_{k}(2 h, h)\left(\frac{x}{1-x}\right)^{2 h}\left((1-x)^{2} w\right)^{h},
$$

which allows for extending the parameter range of $h$

$$
\sum_{n \geq 0} \sum_{h=0}^{\left\lfloor\frac{n}{2}\right\rfloor} C_{k}(n, h) w^{h} x^{n}=\sum_{h \geq 0} \sum_{s=2 h}^{4 h+1} j_{k}(s, h)\left(\frac{x}{1-x}\right)^{s}\left((1-x)^{2} w\right)^{h} .
$$

Setting $u=(1-x)^{2} w$ and $z=\frac{x}{1-x}$, we obtain the bivariate generating function

$$
\sum_{h \geq 0} \sum_{s=2 h}^{4 h+1} j_{k}(s, h) z^{s} u^{h}=\sum_{n \geq 0} \sum_{h=0}^{\left\lfloor\frac{n}{2}\right\rfloor} C_{k}(n, h)\left(u(1+z)^{2}\right)^{h}\left(\frac{z}{1+z}\right)^{n} .
$$

We next consider two power series relations due to [10] and [11]

$$
\begin{aligned}
\sum_{n \geq 0} \sum_{h=0}^{\left\lfloor\frac{n}{2}\right\rfloor} \mathrm{T}_{k, 1}(n, h) v^{h} y^{n} & =\frac{1}{v y^{2}-y+1} \mathbf{F}_{k}\left(\frac{v y^{2}}{\left(v y^{2}-y+1\right)^{2}}\right) \\
\sum_{n \geq 0} \sum_{h=0}^{\left\lfloor\frac{n}{2}\right\rfloor} \mathrm{T}_{k, 1}(n, h) v^{h} y^{n} & =\sum_{n \geq 0} \sum_{h=0}^{\left\lfloor\frac{n}{2}\right\rfloor} C_{k}(n, h)\left(\frac{v}{1-v y^{2}}\right)^{h} y^{n} .
\end{aligned}
$$

In view of eq. (36) and eq. (37), we can conclude

$$
\sum_{h \geq 0} \sum_{s=2 h}^{4 h+1} j_{k}(s, h) z^{s} u^{h}=\frac{(1+z)\left(1+u z^{2}\right)}{u z^{3}+2 u z^{2}+1} \mathbf{F}_{k}\left(\frac{(1+z)^{2}\left(1+u z^{2}\right) u z^{2}}{\left(u z^{3}+2 u z^{2}+1\right)^{2}}\right)
$$


and in particular, setting $u=1$,

$$
\mathbf{J}_{k}(z)=\frac{(1+z)\left(1+z^{2}\right)}{z^{3}+2 z^{2}+1} \mathbf{F}_{k}\left(\frac{(1+z)^{2}\left(1+z^{2}\right) z^{2}}{\left(z^{3}+2 z^{2}+1\right)^{2}}\right)
$$

whence assertion (a).

Assertion (b) follows in complete analogy to the proof of Theorem 3.2. First we verify that the factor $\frac{(1+z)\left(1+z^{2}\right)}{z^{3}+2 z^{2}+1}$ does not introduce a dominant singularity of $\mathbf{J}_{k}(z)$. Then we verify, using Tab. 1, that the unique dominant singularity of $\mathbf{F}_{k}\left(\frac{(1+z)^{2}\left(1+z^{2}\right) z^{2}}{\left(z^{3}+2 z^{2}+1\right)^{2}}\right)$ is the minimum positive real solution of $\frac{(1+z)^{2}\left(1+z^{2}\right) z^{2}}{\left(z^{3}+2 z^{2}+1\right)^{2}}=$ $\rho_{k}^{2}$ for $2 \leq k \leq 9$. Now (b) follows from Proposition 1 .

We finally compute the number of $\operatorname{Iv}_{k}^{1}$-shapes induced by $k$-noncrossing, $\sigma$-canonical RNA structures of fixed length $n, \operatorname{lv}_{k, \sigma}^{1}(n)$, setting

$$
\mathbf{L v}_{k, \sigma}^{1}(x)=\sum_{n \geq 0} \mathbf{I v}_{k, \sigma}^{1}(n) x^{n}
$$

Theorem 4.2. Let $k, \sigma \in \mathbb{N}$, where $k \geq 2$. Then the following assertions hold

(a) the generating function $\mathbf{L} \mathbf{v}_{k, \sigma}^{1}(x)$ is given by

$$
\mathbf{L v}_{k, \sigma}^{1}(x)=\frac{(1+x)\left(1+x^{2 \sigma}\right)}{(1-x)\left(x^{2 \sigma+1}+2 x^{2 \sigma}+1\right)} \mathbf{F}_{k}\left(\frac{(1+x)^{2} x^{2 \sigma}\left(1+x^{2 \sigma}\right)}{\left(x^{2 \sigma+1}+2 x^{2 \sigma}+1\right)^{2}}\right)^{n} .
$$

(b) for $2 \leq k \leq 9$ and $1 \leq \sigma \leq 10$, we have

$$
\operatorname{Iv}_{k, \sigma}^{1}(n) \sim c_{k, \sigma}^{\prime} n^{-\left((k-1)^{2}+(k-1) / 2\right)}\left(\chi_{k, \sigma}^{-1}\right)^{n},
$$

where $c_{k, \sigma}^{\prime}>0$ and $\chi_{k, \sigma}$ is the unique minimum positive real solution of

$$
\frac{(1+x)^{2} x^{2 \sigma}\left(1+x^{2 \sigma}\right)}{\left(x^{2 \sigma+1}+2 x^{2 \sigma}+1\right)^{2}}=\rho_{k}^{2} .
$$

Proof. Obviously, we can inflate any structure by adding arcs into its stacks or duplicating isolated vertices without changing its $\operatorname{Iv}_{k}^{1}$-shape. As a result, we can derive from any $\mathrm{Iv}_{k}^{1}$-shape by inflating its stacks to $\sigma$ arcs, a unique, minimal, $k$-noncrossing, $\sigma$-canonical structure inducing it. This observation implies

$$
\operatorname{Iv}_{k, \sigma}^{1}(n)=\sum_{h=0}^{\left\lfloor\frac{n}{2 \sigma}\right\rfloor \min \{4 h+1, n-2(\sigma-1) h\}} \sum_{s=2 h} j_{k}(s, h)
$$




\begin{tabular}{cccccccc}
\hline$\sigma / k$ & 2 & 3 & 4 & 5 & 6 & 7 & 8 \\
\hline 1 & 2.09188 & 4.51263 & 6.65586 & 8.73227 & 10.7804 & 12.8137 & 14.8381 \\
2 & 1.56947 & 2.31767 & 2.81092 & 3.21184 & 3.55939 & 3.87079 & 4.15552 \\
3 & 1.38475 & 1.80408 & 2.05600 & 2.24968 & 2.41081 & 2.55050 & 2.67477 \\
\hline
\end{tabular}

Table 3: The exponential growth rates $\chi_{k, \sigma}^{-1}$ of $\operatorname{lv}_{k}^{1}$-shapes induced by $k$-noncrossing, $\sigma$-canonical RNA structures of length $n$.

whence we can rewrite the generating function

$$
\mathbf{L v}_{k, \sigma}^{1}(x)=\sum_{h \geq 0} \sum_{s=2 h}^{4 h+1} \sum_{n \geq 2 h(\sigma-1)+s} j_{k}(s, h) x^{n}=\frac{1}{1-x} \sum_{h \geq 0} \sum_{s=2 h}^{4 h+1} j_{k}(s, h) x^{2 h(\sigma-1)+s} .
$$

Employing eq. (32), we derive

$$
\mathbf{L v}_{k, \sigma}^{1}(x)=\frac{(1+x)\left(1+x^{2 \sigma}\right)}{(1-x)\left(x^{2 \sigma+1}+2 x^{2 \sigma}+1\right)} \mathbf{F}_{k}\left(\frac{(1+x)^{2} x^{2 \sigma}\left(1+x^{2 \sigma}\right)}{\left(x^{2 \sigma+1}+2 x^{2 \sigma}+1\right)^{2}}\right)^{n}
$$

and assertion (a) follows. As for assertion (b), we proceed in analogy to the proof of Theorem 3.2 and verify that for $2 \leq k \leq 9$ and $1 \leq \sigma \leq 10$, the unique minimum positive real solution, $\chi_{k, \sigma}$, of eq. (41) is the unique dominant singularity of generating function $\mathbf{L v}_{k, \sigma}^{1}(x)$. Consequently, Proposition 1 implies that

$$
\operatorname{Iv}_{k, \sigma}^{1}(n) \sim c_{k, \sigma}^{\prime} n^{-\left((k-1)^{2}+(k-1) / 2\right)}\left(\chi_{k, \sigma}^{-1}\right)^{n},
$$

where $c_{k, \sigma}^{\prime}$ is some positive constant, whence (b) and the theorem is proved.

\section{Conclusion}

$\mathrm{IV}_{k^{-}}^{1}$ and $\mathbf{I V}_{k}^{5}$-shapes of $k$-noncrossing, $\sigma$-canonical RNA pseudoknot structures provide a significant simplification of complicated molecular configurations with cross-serial interactions. The asymptotic formulas presented in Theorem 3.2 and Theorem 4.2

$$
\begin{aligned}
& \operatorname{Iv}_{k, \sigma}^{5}(n) \sim c_{k, \sigma} n^{-\left((k-1)^{2}+(k-1) / 2\right)}\left(\zeta_{k, \sigma}^{-1}\right)^{n} \\
& \operatorname{lv}_{k, \sigma}^{1}(n) \sim c_{k, \sigma}^{\prime} n^{-\left((k-1)^{2}+(k-1) / 2\right)}\left(\chi_{k, \sigma}^{-1}\right)^{n},
\end{aligned}
$$


imply all asymptotic results on abstract shapes of secondary structures in the literature (note $n^{-\left((k-1)^{2}+(k-1) / 2\right)}=n^{-3 / 2}$ ).

The growth rates of $\mid \mathrm{v}_{k^{-}}^{1}$ and $\mathrm{Iv}_{k^{5}}^{5}$-shapes of $k$-noncrossing, $\sigma$-canonical structures, are displayed in Tab. 4 and Tab. 5, where they are contrasted with the exponential growth rates of $k$-noncrossing, $\sigma$-canonical structures, $\gamma_{k, \sigma}$.

\begin{tabular}{cccccccc}
\hline$k$ & 2 & 3 & 4 & 5 & 6 & 7 & 8 \\
\hline$\gamma_{k, 2}^{-1}$ & 1.96798 & 2.58808 & 3.03825 & 3.41383 & 3.74381 & 4.04195 & 4.31617 \\
$\chi_{k, 2}^{-1}$ & 1.56947 & 2.31767 & 2.81092 & 3.21184 & 3.55939 & 3.87079 & 4.15552 \\
$\zeta_{k, 2}^{-1}$ & 1.26585 & 1.93496 & 2.41152 & 2.80275 & 3.14338 & 3.44943 & 3.72983 \\
\hline
\end{tabular}

Table 4: The exponential growth rates of arbitrary $k$-noncrossing, 2 -canonical RNA structures of length $n$ and the numbers of their induced $I v_{k}^{1}$ and $I v_{k}^{5}$ shapes.

\begin{tabular}{cccccccc}
\hline$k$ & 2 & 3 & 4 & 5 & 6 & 7 & 8 \\
\hline$\gamma_{k, 3}^{-1}$ & 1.71599 & 2.04771 & 2.27036 & 2.44664 & 2.59554 & 2.72590 & 2.84267 \\
$\chi_{k, 3}^{-1}$ & 1.38475 & 1.80408 & 2.05600 & 2.24968 & 2.41081 & 2.55050 & 2.67477 \\
$\zeta_{k, 3}^{-1}$ & 1.17928 & 1.55752 & 1.80082 & 1.98945 & 2.14693 & 2.28376 & 2.40567 \\
\hline
\end{tabular}

Table 5: The exponential growth rates of arbitrary $k$-noncrossing, 3 -canonical RNA structures of length $n$ and the numbers of their induced $I_{k}^{1}$ and $I_{k}^{5}$ shapes.

Table 5 shows that the exponential growth rate of $\mathrm{lv}_{3}^{5}$-shapes of $k$-noncrossing 3 -canonical structures are significantly smaller than that of all $k$-noncrossing 3 -canonical structures. Therefore, the abstract $I v_{3}^{5}$-shapes represent a meaningful reduction. At http://www.combinatorics.cn/cbpc/paper.html, we provide supplemental material for our results.

Acknowledgments. This work was supported by the 973 Project, the PCSIRT Project of the Ministry of Education, the Ministry of Science and Technology, and the National Science Foundation of China. 


\section{References}

[1] M. Chamorro, N. Parkin, H.E. Varmus, An RNA pseudoknot and an optimal heptameric shift site are required for highly efficient ribosomal frameshifting on a retroviral messenger RNA, J. Proc. Natl. Acad. Sci. USA 89 (1991) 713-717.

[2] W.Y.C. Chen, E.Y.P. Deng, R.R.X. Du, R.P. Stanley, C.H. Yan, Crossings and nestings of matchings and partitions, Trans. Amer. Math. Soc. 359(4) (2007) 1555-1575.

[3] P. Flajolet, R. Sedgewick, Analytic combinatorics, Cambridge University Press, New York, 2009.

[4] R. Giegerich, B. Voß, M. Rehmsmeier, Abstract shapes of RNA, Nucleic Acids Res. 32 (2004) 4843-4851.

[5] D.J. Grabiner, P. Magyar, Random walks in Weyl chambers and the decomposition of tensor powers, J. Algebr. Comb. 2 (1993) 239-260.

[6] I.L. Hofacker, P. Schuster, P.F. Stadler, Combinatorics of RNA secondary structures., Discr. Appl. Math. 88 (1998) 207-237.

[7] I.L. Hofacker, Vienna RNA secondary structure server, Nucl. Acids. Res. 31(13) (2003) 3429-3431.

[8] F.W.D. Huang, W.W.J. Peng, C.M. Reidys, Folding 3-noncrossing RNA pseudoknot structures, J. Comput. Biol. (2009) to appear.

[9] E.Y. Jin, J. Qin, C.M. Reidys, Combinatorics of RNA structures with pseudoknots, Bull. Math. Biol. 70(1) (2008) 45-67.

[10] E.Y. Jin, C.M. Reidys, Asymptotic enumeration of RNA structures with pseudoknots, Bull. Math. Biol. 70(4) (2008) 951-970.

[11] E.Y. Jin, C.M. Reidys, Combinatorial design of pseudoknot RNA, Adv. Appl. Math. 42 (2009) 135-151.

[12] E.Y. Jin, C.M. Reidys, R.R. Wang, Asympotic analysis of $k$-noncrossing matchings, arXiv:0803.0848, (2008). 
[13] D. Kleitman, Proportions of irreducible diagrams, Studies in Appl. Math. 49 (1970) 297-299.

[14] D.A.M. Konings, R.R. Gutell, A compariosn of thermodynamic folidngs with comparatively derived structures of $16 \mathrm{~S}$ and $16 \mathrm{~S}$-like rRNAs, RNA 1 (1995) 559-574.

[15] A. Loria, T. Pan, Domain structure of the ribozyme from eubacterial ribonuclease P, RNA 2 (1996) 551-563.

[16] W.A. Lorenz, Y. Ponty, P. Clote, Asymptotics of RNA shapes, J. Comput. Biol. 15(1) (2008) 31-63.

[17] R.B. Lyngso, C.N.S. Pedersen, RNA pseudoknot prediction in energybased models, J. Comput. Biol. 7 (2000) 409-427.

[18] G. Ma, C.M. Reidys, Canonical RNA pseudoknot structures, J. Comput. Biol. 15(10) (2008) 1257-1273.

[19] J.S. McCaskill, The equilibrium partition function and base pair binding probabilities for RNA secondary structure, Biopolymers 29 (1990) 11051119 .

[20] Mapping RNA form and function, Sicence 309(5740) (2005) 1441-1632.

[21] M.E. Nebel, A. Scheid, On quantitative effects of RNA shape abstraction, Theory in Biosciences, to appear.

[22] R. Nussinov, G. Pieczenik, J.R. Griggs, D.J. Kleitman, Algorithms for loop matchings, SIAM J. of Appl. Math. 35 (1978) 68-82.

[23] E. Rivas, S.R. Eddy, A dynamic programming algorithm for RNA structure prediction including pseudoknots, J. of Mol. Biol. 285(5) (1999) 2053-2068.

[24] R. Stanley, Differentiably finite power series, Europ. J. Combinatorics 1 (1980) 175-188.

[25] D.W. Staple, S.E. Butcher, Pseudoknots: RNA structures with diverse functions, PLoS Biol. 3(6) (2005) 956-959. 
[26] E.C. Titchmarsh, The theory of functions, Oxford Uninversity Press, Oxford, UK, 1939.

[27] C. Tuerk, S. MacDougal, L. Gold, RNA pseudoknots that inhibit human immunodeficiency virus type 1 reverse transcriptase, Proc. Natl. Acad. Sci. USA 89 (1992) 6988-6992.

[28] B. Voß, R. Giegerich, M. Rehmsmeier, Complete probabilistic analysis of RNA shapes, BMC Biology 4(5) (2006) 1-23.

[29] W. Wasow. Asymptotic expansions for ordinary differential equations, Dover, New York, 1987.

[30] M.S. Waterman, Secondary structure of single-stranded nucleic acids, Adv. Math.I (suppl.) 1 (1978) 167-212.

[31] M.S. Waterman, Combinatorics of RNA hairpins and cloverleafs, Stud. Appl. Math. 60 (1979) 91-96.

[32] M.S. Waterman, W.R. Schmitt, Linear trees and RNA secondary structure, Discr. Appl. Math. 51 (1994) 317-323.

[33] E. Westhof, L. Jaeger, RNA pseudoknots, Curr. Opin. Struct. Biol. 2 (1992) 327-333. 